NFSA/TM-CYO 207069

\title{
RAPID TEMPORAL CHANGES OF MIDTROPOSPHERIC WINDS N/S
}

$$
\begin{aligned}
& \text { NSB UASA } \\
& \text { NNY\%- TP } \\
& \text { (c) wHite } \\
& 067185
\end{aligned}
$$

Francis J. Merceret

Printed in the United Stales of America Reprinted from Journal of Applied Meteorology

Vol. 36, No. 11, November 1997

( 1997 American Meteorological Society 


\title{
Rapid Temporal Changes of Midtropospheric Winds
}

\author{
FRANCIS J. MERCERET \\ Applied Meteorology Lnit, NASA/Konnedy Space Center, Florida \\ (Manuscript received 7 October 1996, in final form 17 April 1997)
}

ABSTRACT

\begin{abstract}
The statistical distribution of the magnitude of the vector wind change over $0.25-., 1-, 2-$, and 4-h periods based on data from Oetober 1995 through March 1996 over central Florida is presented. The wind changes at altitudes from 6 to $17 \mathrm{~km}$ were measured using the Kennedy Space Center 50-MHz Doppler radar wind profiler. Quality controlled profiles were produced every $5 \mathrm{~min}$ for 112 gates, each representing $150 \mathrm{~m}$ in altitude. Gates 28 through 100 were selected for analysis because of their significance to ascending space launch vehicles. The distribution was found to be lognormal. The parameters of the lognormal distribution depend systematically on the time interval. This dependence is consistent with the behavior of structure functions in the $f^{5 / 4}$ spectral regime. There is a small difference between the 1995 data and the 1996 data, which may represent a weak seasonal effect.
\end{abstract}

\section{Introduction}

This study was motivated by the need to quantify the benefit of continuing to operate the Kennedy Space Center (KSC) $50-\mathrm{MHz}$ Doppler radar wind profiler (DRWP). In its operational support configuration, the instrument measures wind speed and direction at $150-\mathrm{m}$ intervals from 2011 to $18661 \mathrm{~m}$. The data are currently used to evaluate wind persistence between the last precision wind sounding balloon ("Jimsphere"), about 1-h before launch, and launch of a space shuttle. Other launch vehicles may soon begin using the DRWP in a similar manner.

The benefit of the DRWP is avoidance of the risk associated with undetected wind changes large enough to threaten the vehicle or the mission. To assist in assessing this risk, the statistical distribution of the vector magnitudes of $0.25-, 1-, 2-$, and $4-\mathrm{h}$ wind changes at altitudes between 6 and $17 \mathrm{~km}$ was determined over a 6 -month period during the winter season. The $6-17-\mathrm{km}$ region is the region in which large launch vehicles are most sensitive to wind changes. Winter is the season in which the largest short-term temporal changes in winds at the target altitudes over Florida are expected. Wind changes rather than wind shear were examined because space vehicle load programs calculate loads based on the wind profiles, and it is the deviation from the expected wind profile that poses the hazard regardless of whether large shears are involved.

Corresponding author address: Dr. Francis J. Merceret, Applied Meteorology Unit-NASA, Mail Code PH-B3, Kennedy Space Center, FL 32899

E-mail: francis.merceret-1 (a)ksc.nasa.gov
Wind profiles were collected every 5 min over a period of 117 days from 29 September 1995 through 26 March 1996 except when the DRWP was down for maintenance or repair. The data were extensively quality controlled. Vector wind differences were computed at each level for the target time intervals $(\Delta T)$ for each profile using only data that passed the quality control (QC) screening. More than 25000 profiles were accepted for analysis. The first four moments, the probability density, and the cumulative probability distribution of the vector magnitude were computed at each level for each $\Delta T$.

This paper presents the details of how the study was conducted and the results showing the statistical distributions of the vector wind changes as a function of $\Delta T$. Results include statistics for the distribution at any one gate in the target region and also for the maximum vector wind change magnitude in the entire region.

\section{The KSC 50-MHz DRWP}

The data used for this study were collected using the 50-MHz DRWP located near the Space Shuttle Landing Facility (SLF). It is a hardware twin to the one at White Sands Missile Range described by Nastrom and Eaton (1995). The consensus software supplied with the original profiler was replaced by a "first-guess median filter" algorithm developed at Marshall Spaceflight Center (Wilfong et al. 1993) that also provides capability for real-time interactive QC of the data. Details are provided in Schumann et al. (1993).

Profiles are generated every $5 \mathrm{~min}$. Each profile contains data at each of 112 range gates that represent a slice of the atmosphere $150 \mathrm{~m}$ thick. Gate 1 is at a 
nominal altitude of $2011 \mathrm{~m}$ while gate 112 is at 18661 $\mathrm{m}$. The variables measured at each gate include the signal power, noise power, Doppler return frequency, and spectral width for each of the two oblique beams and the vertical beam. These data are converted into estimates at each gate of the horizontal wind direction and speed, the vertical speed, and the signal-to-noise ratio in each beam.

\section{The data and its analysis}

a. The data sample

At each of the 112 range gates, the KSC DRWP produces 288 wind speed and direction values daily. Data were collected for this study from 29 September 1995 through 26 March 1996. Excluding days on which the DRWP was inoperative, there were 61 days in 1995 and 56 days in 1996 that yielded 117 days in the sample. Some of these days were incomplete due to profiler outages, and the QC process removed additional data. Since the QC was done gate by gate, the sample size varies; but it is typically 25000 to 28000 per gate. When data are combined for gates $28-100(6-17 \mathrm{~km})$, the resulting sample size approaches two million.

The time available for this study was limited and a full year of data could not be collected or processed. The upper air climatology of central Florida is such that the winter season brings stronger winds aloft, including meandering jet cores with sharp spatial gradients. This season was selected for the study as a "worst case" in terms of the risk to space launch vehicles. Obviously, this is a worst case only in the sense of being worse than the summer season. It is unlikely to be the worst case over a period of years.

To estimate the strength of seasonal variation, the sample was nearly evenly divided, with the first half from 1995 and the second from 1996. Typically, central Florida winds aloft and their gradients are somewhat stronger in the January through March period than in October through December. The mean layer wind speeds for gates 28-100 followed the typical pattern. For 29 September through 31 December 1995 the dataset gave a mean speed of $23.19 \mathrm{~m} \mathrm{~s}$. For the data from 1 January through 26 March 1996 the result was $35.46 \mathrm{~m}$ $\mathrm{s}$. The respective standard deviations ( $\mathrm{m} \mathrm{s}{ }^{1}$ ) were 13.66 and 14.31. The sample size for both sets was near a million, and both of the differences are statistically significant at the 0.005 level. Comparison of the 1995 and 1996 results with each other and with the combined 1995-96 data was used to examine whether a seasonal effect was significant during these months.

\section{b. The quality control (QC) process}

Each range gate in each profile constitutes one record in the daily data files. Each record includes a QC flag to track individual quality control tests. The active flag bits are listed below in Table 1.
TABL.F 1. Quality control flag thresholds

\begin{tabular}{|c|c|}
\hline What causes flag & $\begin{array}{c}\text { Threshold } \\
\text { valus }\end{array}$ \\
\hline $\begin{array}{l}\text { Excessive shear internally computed by } \\
\text { DRWP }\end{array}$ & $0.1 ; 1$ \\
\hline Excessive vertical speed & $3 \mathrm{~m} \times 1$ \\
\hline Excessive spectral width & $3 \mathrm{~m} \times 1$ \\
\hline Exceeds first-guess propagation limit & 6 \\
\hline Missing profile-data place holders (999) & $\mathrm{N} / \mathrm{A}$ \\
\hline Fails small median test & See text \\
\hline Excessive adjacent gate directional shear & 30 per gate \\
\hline Excessive adjacent gate speed shear & $7 \mathrm{~m} s \mathrm{per}$ galc \\
\hline Insutficient signal-to-noise ratio (SNR) & $-15 \mathrm{~dB}$ \\
\hline Fails manual QC & See $\mid \mathrm{exl}$ \\
\hline
\end{tabular}

The vertical speed threshold is used to identify precipitation. Heavy rain contaminates the radar signal and produces bad winds. Typically, heavy rain produces a large vertical wind indication based on the radar tracking the falling raindrops.

The shear value computed internally by the radar software provides a coarse warning that the measurements are invalid and would usually not be triggered except by an aircraft in the beam or a major failure in the system. The more stringent speed and direction shear tests are designed to eliminate meteorologically unrealistic winds produced by sidelobe or interference signals and to flag those signals.

The small median test is the one described in detail by Carr et al. (1995). It examines not only continuity of the winds in the vertical but temporal continuity as well. The test requires threshold values be designated for three different heights. This work used $5.7 \mathrm{~m} \mathrm{~s}{ }^{\prime}$ at $2 \mathrm{~km}, 10.2 \mathrm{~m} \mathrm{~s}^{\prime}$ at $9 \mathrm{~km}$, and $8.4 \mathrm{~m} \mathrm{~s}{ }^{\prime}$ at $16 \mathrm{~km}$ based on about 20 years of local windsonde data. These criteria are more restrictive than those used by Carr et al. (1995).

On many of the days during the experimental period, personnel of the Applied Meteorology Unit (AMU) examined the spectra in real time and made notes of any obvious sidelobe or interference signals that could adversely affect the data. When the Titan program conducted real or simulated launch operations, the data were not only monitored but were manually quality controlled before transmission to the data system. In these cases, the real-time manual QC operator made extensive notes. Both the AMU notes and the Titan notes were used to assist the after-the-fact manual QC process described next.

After the automated QC was run, the file was displayed graphically. Wind speed and wind direction were separately but simultaneously presented as time-height diagrams in which each range gate at each 5-min interval was plotted as a colored point. The resulting color contour map permitted visual detection of wind speed or wind direction changes and the identification of natural features like jet streaks and artifacts like sidelobes.

AMU and Titan QC logs were used for guidance. During visual inspection of the data, the time and al- 


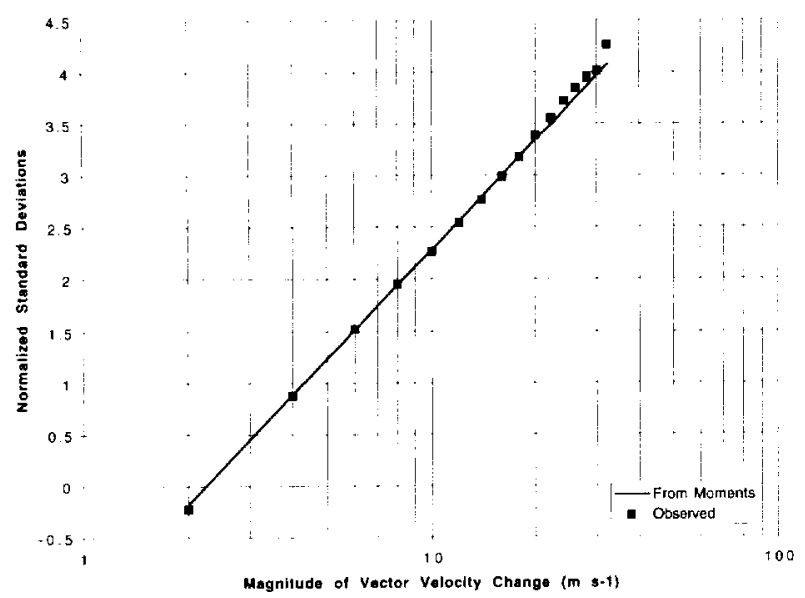

FIG. 1. The cumulative probability (vertical axis) of the magnitude of the 1-h wind change at any level in the region from gate 28 to gate 100 inclusive exceeding the specified (horizontal axis) value. Probabilities are expressed as normalized standard deviations z (e.g. a probability of 0.5 equates to $z=0,0.8413$ equates to $z=1$ ). The points are the measured cumulative distribution. The line is the lognormal distribution derived from the moments as deseribed in the text.

titude of any apparent sidelobe or interference signatures were noted. Data that appeared to be unreliable were manually flagged by setting a QC flag bit.

Nearly all of the manual QC consisted of flagging the interior (in time-height space) of sidelobe and interference signatures whose boundaries were flagged by the automated process. Of the 117 days in the sample, 44 required some manual flagging. Less than $1 \%$ of those data were manually flagged.

Once a data file had been accepted, the delta files were generated and examined visually. Delta files are identical to the data files except that they contain the wind changes rather than the winds. Sometimes a sidelobe or interference signal stood out better in the wind changes than in the winds themselves. When this occurred, the process described above was repeated. When the data file had been reaccepted, the delta files were rerun and reexamined. The data were used for analysis only when the basic data and the delta files had been examined and accepted. During analysis, any record for which the QC flag is nonzero was ignored.

\section{c. The analysis scheme}

The initial experimental design called for computing the first four statistical moments (mean, variance, skewness, and kurtosis) and the probability density and distribution of the magnitude of the vector wind change at each level in the region of interest. It became quickly obvious that this was impractical. Greater sample sizes with all of their advantages for statistical analysis could be obtained by combining data from multiple levels while simultaneously reducing the information output to a manageable size. Limited resources made this strategy nec- essary despite the loss of information about the variability of the results with height. The height levels from gate 28 to gate 100 were selected for their relevance to ascending launch vehicles. In addition, the maximum value for the magnitude of the vector wind change anywhere in the target region was computed for each profile. The same statistics were computed for this dataset.

A combination of gates 32-34 (a shuttle's transonic region) was created to permit "quick-look" examination of the extent to which vertical inhomogeneity might be a concern. The mean wind speed in this layer averaged $30 \%$ lower than in the layer from gate 28 to gate 100 . The wind difference data from the two regions differed by an amount that was statistically significant due to the enormous sample sizes involved, but the differences appeared insignificant to the author from the point of view of risk assessment. After determining that the distribution of these data had roughly the same shape and parameter values as those in the thicker layer, detailed analysis of this dataset was deferred due to limited resources. Limited resources also prevented any attempt to stratify the data temporally to look for diurnal effects.

Examination of the statistical results from the first two days of the September 1995 data suggested that the data were distributed lognormally. The experimental design was enhanced to include generation of six estimates of the parameters of a lognormal distribution from the four moments by combining them in pairs. Each pair of moments generates an estimate for the two lognormal parameters as shown below. The mutual consistency of these estimates served as an indicator that the distributions really were lognormal. This was confirmed by using the mean of the six estimates of each parameter to generate a straight line on a log probability plot on which the actual distribution was overlaid for comparison.

The formula for the $n$th central moment of a lognormal distribution is given by Aitchison and Brown (1966) as

$$
M^{n}=\exp \left(n \mu+n^{2} \sigma^{2} / 2\right),
$$

where $\mu$ is the mean of the logarithms and $\sigma$ is the standard deviation of the logarithms of the lognormal variable. For any two values of $n$ for which $M^{n}$ are known, the result is a pair of simultaneous linear algebraic equations for $\mu$ and $\sigma^{2}$, which may be solved readily.

\section{The results}

This section presents the analysis showing that the magnitude of the vector wind change is lognormally distributed. The variation of the parameters of the lognormal distribution with the length of the time interval over which the change takes place is also presented. A brief discussion of possible seasonal variation in the distribution is included. Finally, the implications of these results for spaceflight operations and for the design of profiler networks are discussed. 


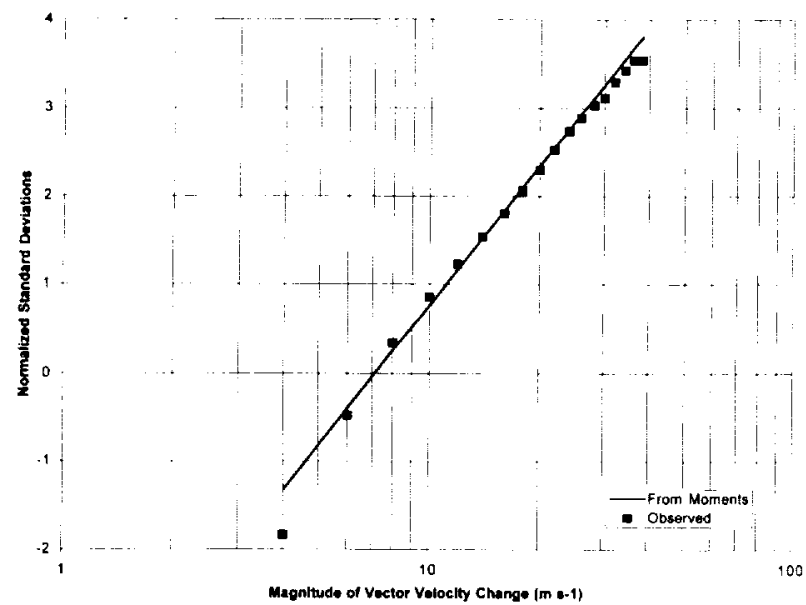

FIG. 2. The cumulative probability of the largest single value of the $\mathrm{I}-\mathrm{h}$ wind change in the region from gate 28 to gate 100 inclusive exceeding the specified value. The format is the same as in Fig. 1.

\section{a. The magnitude of the vector wind change is lognormally distributed}

Figure 1 shows the cumulative distribution of the combined gates 28-100 data for the entire sample of 1 - $\mathrm{h}$ wind changes. The individually plotted points are the actual measured distribution. The straight line represents a pure lognormal distribution with parameters equal to the average of those derived from the six pairwise combinations of the first four moments of the measured distribution. The horizontal axis is the magnitude of the vector wind change over a 1 -h period. This sample contained 1882502 data points. To the extent that the wind differences may be related to wind variances, these results agree with those of Nastrom and Eaton (1995), who found the variances over a 1-h period at similar altitudes were lognormally distributed.

Figure 2 shows the same information as Fig. 1 except that the data are the maximum values of the magnitude of the vector wind change in gates 28-100 for each profile rather than the individual gate values. The sample size is 25983 . This figure shows that the maximum values in the entire region are also lognormally distributed.

The data from 1995 and 1996 separately and the individual monthly data behave in the same manner, as do the data for time periods of $0.25,2$, and $4 \mathrm{~h}$. Figures 1 and 2 above are typical of any of these data subsets.

\section{$b$. The parameters of the lognormal distribution depend on $\Delta \mathrm{T}$}

The two parameters of the lognormal distribution, $\mu$ and $\sigma$, were computed for velocity magnitudes in meters per second. Figure 3 shows the variation of $\mu$ with the time over which the changes are measured $(\Delta T)$ for the gates $28-100$ combined data. Figure 4 shows the variation of $\mu$ for the layer maxima. Despite the significant scatter, a clear, roughly linear relation between the value of $\mu$ and the logarithm of $\Delta T$ is apparent in both cases. Linear regressions of $\mu$ versus $\ln (\Delta T)$ yield $r^{2}>0.9$ (significant to $<0.005$ ) in all cases.

Figures 5 and 6 present the same information for $\sigma$.

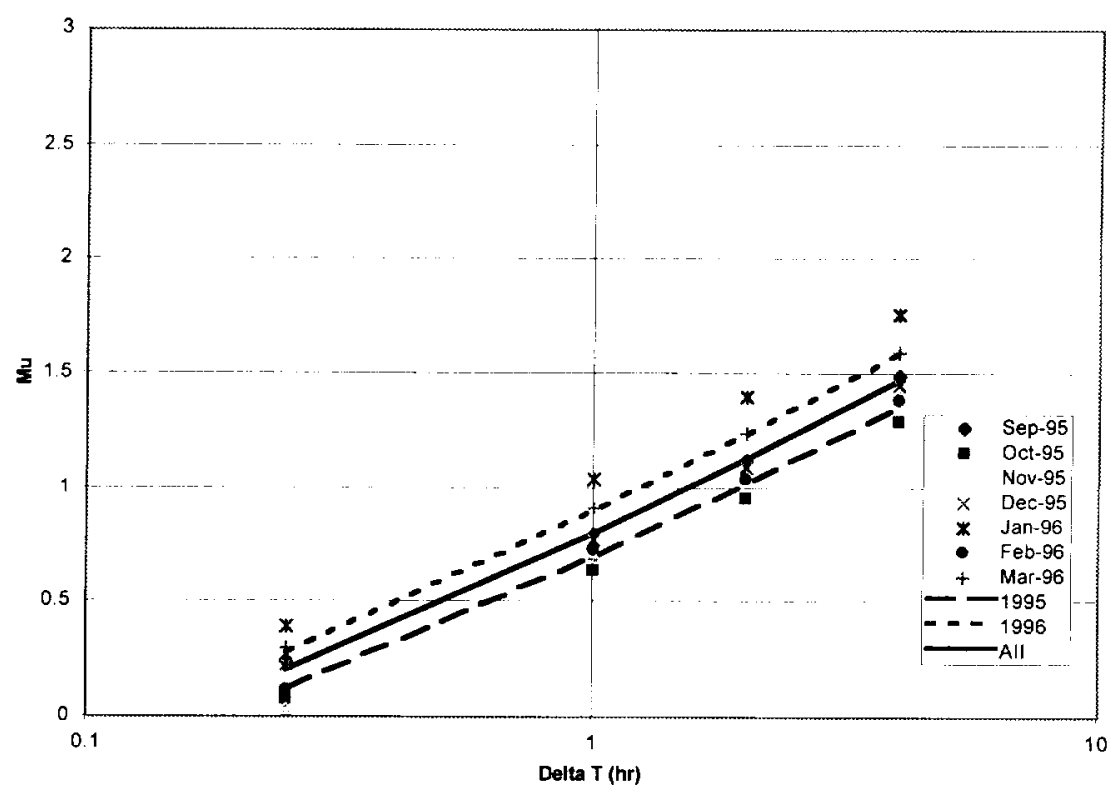

FIG. 3. The relationship between the mean $(\mu)$ of the natural logarithm of the magnitude of the vector wind change ( $\mathrm{m} \mathrm{s}^{1}$ ) change at any height level in the region from gate $28 \mathrm{to}$ gate 100 inclusive and the time interval $(\Delta T)$ over which the change takes place. The lines represent combined data from September-December 1995, January-March 1996, and the entire datiset. The points represent individual months to show the scatter. 


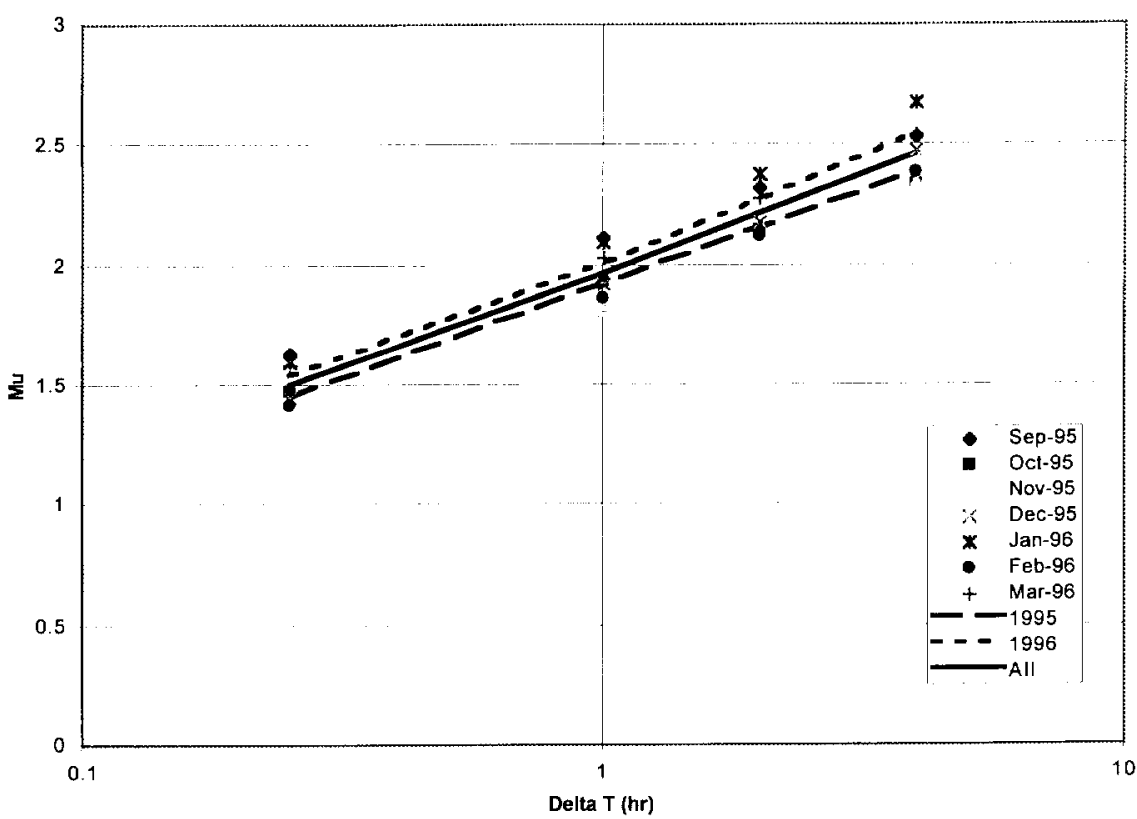

FIG 4 . The relationship between the mean $(\mu)$ of the natural logarithm of the magnitude of the Jargest single vector wind change $(\mathrm{m} \mathrm{s}$ ) change in the region from gate 28 to gate 100 inclusive and the time interval $(\Delta T)$ over which the change takes place. The format is the same as in Fig. 3 .

Although the scatter is much larger, a similar relation with opposite sign is suggested. Linear regressions of $\sigma$ versus $\ln (\Delta T)$ yield $r^{2}>0.4$ (also significant to $<0.005$ ) in all cases.

The relationships between the lognormal parameters and $\Delta T$ can be used to interpolate these results to any
$\Delta T$ between 0.25 and $4 \mathrm{~h}$. They can also be used with appropriate caution to extrapolate these results to larger and smaller time intervals. Extrapolation to larger time intervals is less justified because semidiurnal, diurnal, and synoptic features should begin to dominate stochastic ones at these scales.

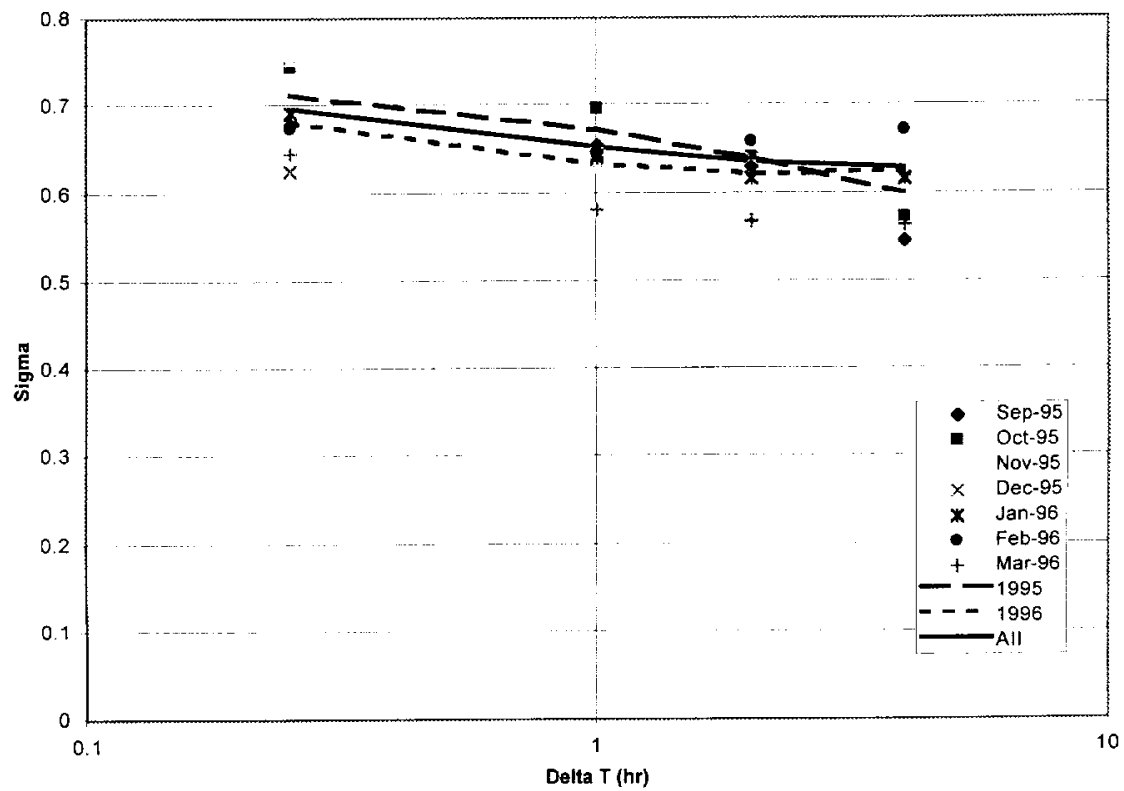

Fig. 5. The relationship between the standard deviation $(\sigma)$ of the natural logarithm of the magnitude of the vector wind change ( $\mathrm{m} \mathrm{s}$ ') change at any height level in the region from gate 28 to gate 100 inclusive and the time interval $(\Delta T)$ over which the change takes place. The format is the same as in Fig. 3. 


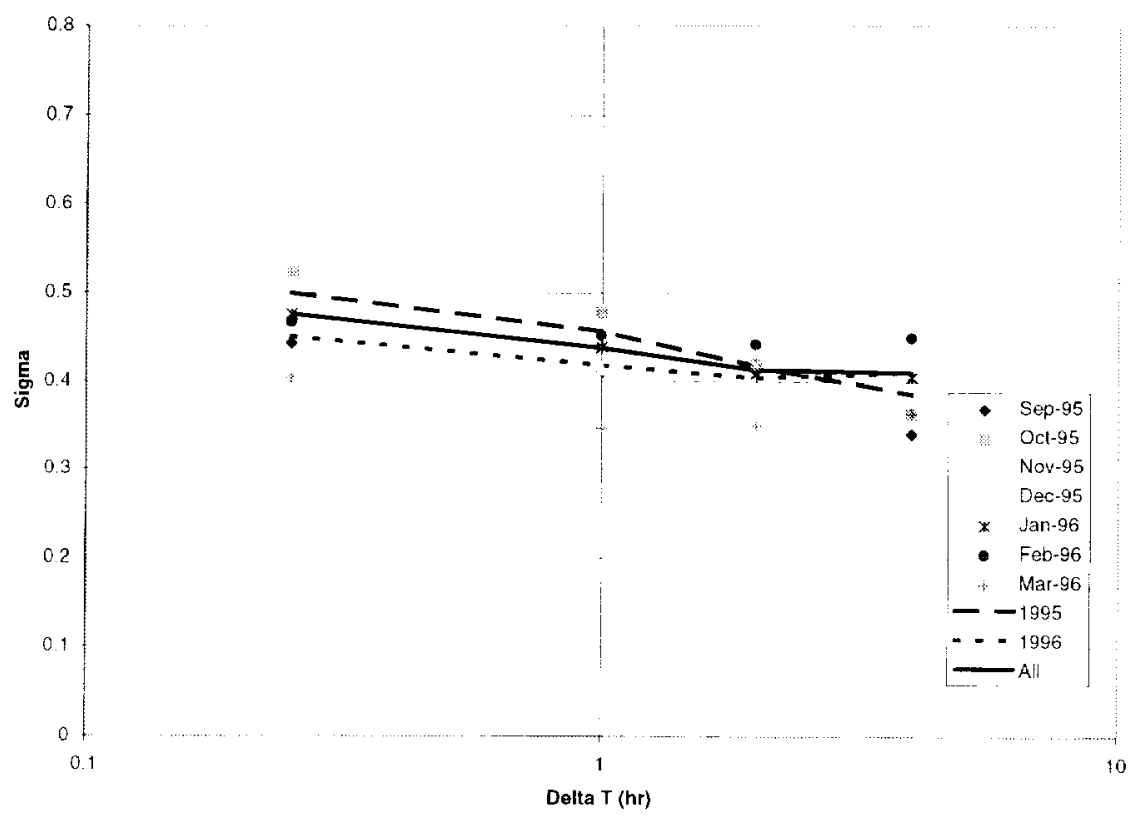

Fig. 6. The relationship between the slandard deviation $(\sigma)$ of the natural logarithm of the magnitude of the largest single vector wind change $(\mathrm{m} \mathrm{s}$ ') change in the region from gate 28 to gate 100 inclusive and the time interval $(\Delta T)$ over which the change takes place. The format is the same as in Fig. 3 .

\section{c. The implications of the distribution}

Examination of the previous figures suggests that part of the scatter may be due to secular changes in the environment during the experimental period. The $\mu$ and $\sigma$ values tend to change in opposite directions with $\Delta T$. The $\mu$ values for 1996 were slightly higher than for 1995, but the $\sigma$ values were lower, thus maintaining the same negative correlation. Although the scatter is large, a $t$ test shows the differences in $\mu$ are statistically significant at the 0.001 level, and a chi-squared test shows that the differences in $\sigma$ are significant at the 0.005 level. In any case, the secular variation is not large compared with the scatter and it remains to be seen whether it is meteorologically significant. These effects, whatever their cause, also naturally appear in the probability of exceedance curves presented in the next section.

The lognormal distribution of the magnitude of the vector wind changes facilitates comparison of these results with atmospheric structure functions. Structure functions for wind are usually defined for each velocity component in terms of spatial separation by or in terms of temporal separation by

$$
D_{u}(r)=\left\langle\left[u_{i}(x)-u_{i}(x+r)\right] "\right\rangle
$$

or in terms of temporal separation by

$$
D_{r i}(\tau)=\left\langle\left[u_{i}(t)-u_{i}(t+\tau)\right]^{2}\right\rangle,
$$

where the brackets denote the average and the subscript $i$ denotes the $i$ th component (Stull 1989).

If the logarithm of the wind differences is normally distributed, then the logarithm of their square is also normally distributed with twice the mean and twice the standard deviation of the distribution of the $\log$ of the differences. If the parameters of the distribution are known as a function of temporal separation, then the mean value of the squared differences can be calculated as a function of the temporal separation. Since the whole vector magnitude was used in this study rather than any single component, the result will be a "magnitude structure function" equal to the sum of the three component structure functions.

The small number of independent estimates of the behavior of the distribution parameters with temporal separation and the scatter in the data suggest that precision calculations are unwarranted. To compare these results with those published in the literature, the variation of $\mu$ and $\sigma$ with $\Delta T$ was approximated by a straight line fit through the 0.25 - and 4 -h values for the combined dataset. This resulted in linear equations for $\mu$ and $\sigma$ as follows:

$$
\begin{aligned}
& 2 \mu=a_{\mu}+b_{\mu} \ln \Delta t \\
& 2 \sigma=a_{i r}+b_{v} \ln \Delta t,
\end{aligned}
$$

where the factor of 2 converts the fit for differences to one for squares of differences. Time differences in seconds were used for dimensional consistency.

The resulting mean value of the squared differences, $D(\Delta t)$, may be reduced to the form

$$
D(\Delta t)=C_{1} \Delta t^{N} \Delta t^{\text {klust, }},
$$

where $C_{1}=\exp \left(a_{\mu}+0.5 a_{i r}^{2}\right), N=b_{\mu}-a_{r} b_{r,}$, and $k=$ $0.5 b_{t}^{2}$. When the data from Figs. 3 and 5 are used to 


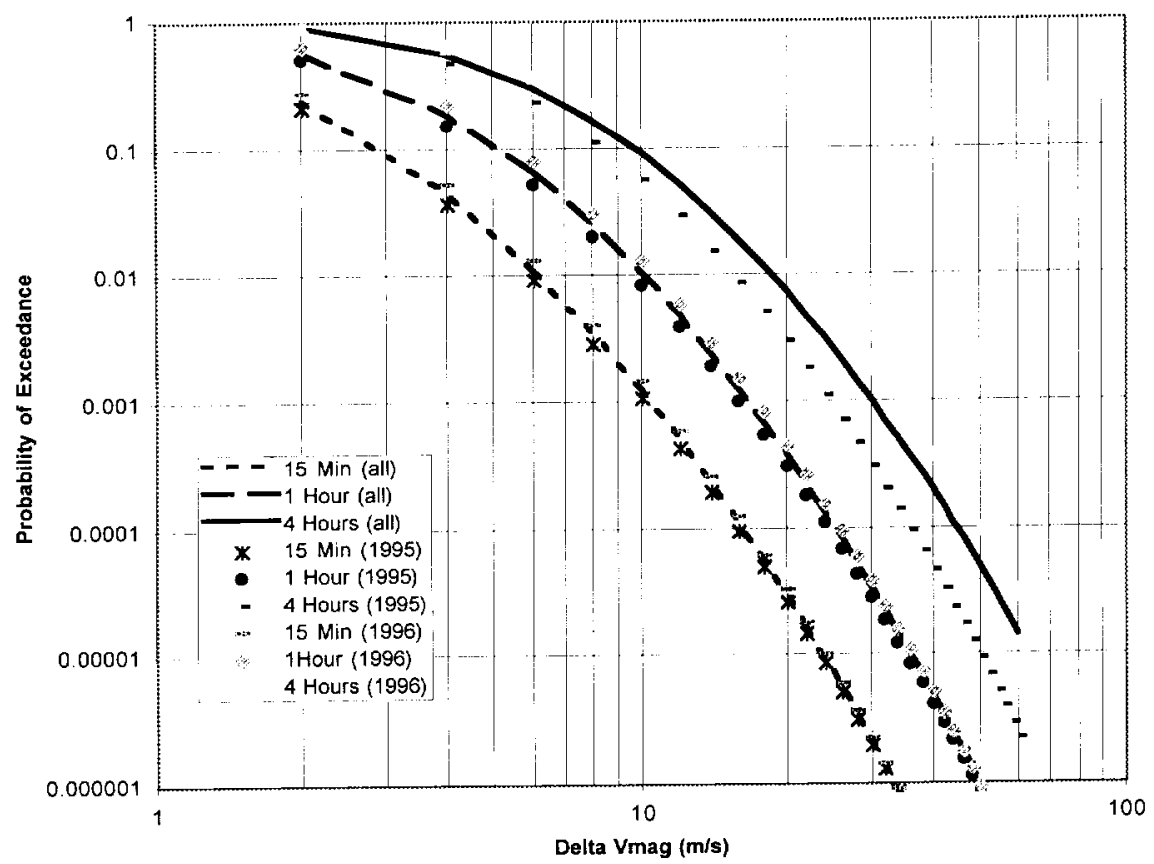

FIG. 7. The probability (vertical axis) of the magnitude of the 1 -h wind change at any height level in the region from gate 28 to gate 100 inclusive exceding the specified (horizontal axis) value for various values of $\Delta T$. The lines are results from the entire dataset. The points separately present the 1995 and 1996 data to show the variation from late fall to early spring.

generate the constants in the linear equations given above, the result is $C=0.01, N=0.8$, and $k=0.001$ to one significant figure.

Over the range from 0.25 - to 4 -h, the term containing $k$ varies from near unity to 1.17 . If we treat this term as approximately constant, the result resembles that widely reported in the literature for structure functions in the inertial subrange if the assumption is made that spatial and temporal variations may be treated similarly using a Taylor's hypothesis. The spatial structure function under these conditions is given by

$$
D(r)=C \varepsilon^{2 / 3} r^{2 / 3}
$$

where $C$ is a constant of order unity (Ottersten 1969). The value of $N$ obtained here is within $20 \%$ of the inertial subrange value. Moreover, the value of $C_{1}$ obtained here implies that $\varepsilon$ is of the order of $0.001 \mathrm{~m}^{2}$ $\mathrm{s} 3$, which compares well with values obtained in the free atmosphere from aircraft observations (Merceret 1976; Vinnichenko and Dutton 1969), although up to two orders of magnitude smaller values have been reported in the upper troposphere and lower stratosphere (Vinnichenko 1970). Significantly, the data obtained here were taken during the winter where both the wind speed and wind shear are greatest, suggesting the largest degree of production and dissipation of mechanically generated turbulence.

No spectral analysis has yet been undertaken with these data, but Vinnichenko (1970) concluded that in the mesoscale (periods from 1 day to about $10 \mathrm{~min}$ ), the $-5 / 3$ power law is followed fairly well. The data here fall within those scales, and the slope of the structure function is consistent with that spectral behavior.

\section{d. Significance for spaceflight operations and the design of upper-air networks}

Using the lognormal model with parameter values based on the data collected in this study, we have generated the required curves for $0.25-, 1-$, and 4 -h intervals. The data for the 2 -h interval were also generated, but are not plotted in the figures to enhance legibility. Figure 7 presents the curves for probability of exceedance at any preselected gate from 28 to 100 . The vertical axis is the probability that the magnitude of the vector wind change over the specified time period will exceed the corresponding value on the horizontal axis. Figure 8 presents the probability that the threshold will be exceeded anywhere in the entire layer.

For spaceflight operations, the benefit of rapid access to wind profile data is the avoidance of unanticipated wind changes, which pose the threat of destruction of a launch vehicle (Mecham 1995). For assessing the hazards to space launch operations, engineers must first determine what wind change between the last balloon and launch poses an operational threat to the launch vehicle. The curves presented here can then be used to quantify the likelihood of that threat. If the risk is unacceptably high, then availability of day of launch DRWP capability should be a mission requirement. A 


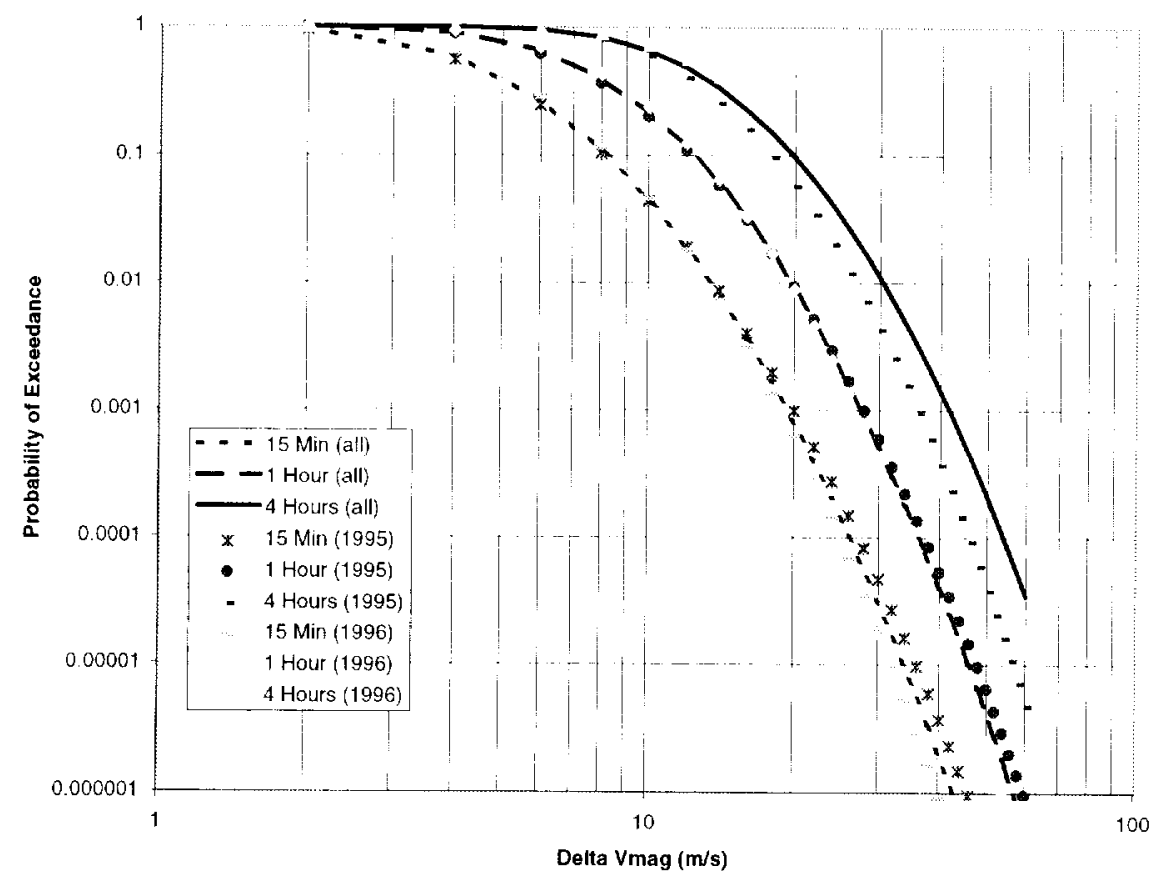

Firi. 8. The probability (vertical axis) of the magnitude of the largest 1-h wind change in the region from gate 28 to gate 100 inclusive exceeding the specified (horizontal axis) value for various values of $\Delta T$. The format is the same as in Fig. 7.

larger dataset and additional analysis could be used to stratify the risk by altitude and season.

For design of upper-air networks, the required temporal sampling rate depends on the need to assume that significant features are observed and the data are representative (Douglas and Stensrud 1996). Sampling rates for current profiler networks are typically one per hour based on consensus averages of 10 or more profiles (NOAA 1994; Spencer et al. 1996). These results allow quantitative assessment of the wind changes that might be missed with sampling or reporting intervals greater than a specified time. This information can be used to optimize the trade-off between cost and complexity of data collection and the accuracy and completeness of the data.

\section{Summary and conclusions}

An examination of six months of winter season $50-\mathrm{MHz}$ DRWP data over central Florida has shown that the magnitude of vector wind changes for periods from 0.25 to $4 \mathrm{~h}$ is lognormally distributed. The lognormal parameters vary systematically and simply with the time interval.

Probability of exceedance curves for wind changes at any single $150-\mathrm{m}$ gate in the region between 6 and $17 \mathrm{~km}$ and also for the maxima within the entire region were also generated. These curves can be used to assess the risk to space launch programs of short period wind changes and to assist in determining the required temporal resolution for proposed profiler networks. They can also be used to assist in the determination of whether the cost of operating a profiler is warranted for a particular application given a specified acceptable risk level.

Acknowledgments. The author thanks Susan DeRussy of Computer Sciences Raytheon for saving the data used in this study. Robin Schumann, Ann Yersavich, and Winnie Lambert of ENSCO, Inc. (AMU), provided the daily logs of profiler performance. Ms. Schumann also wrote the software that transferred the MIDDS data from magnetic tape to $\mathrm{PC}$ readable diskette and confirmed file integrity and format. Ms. Yersavich ran the software to transfer and process the data to readable diskette. Captain Scot Heckman of the 45th Weather Squadron performed some of the real-time QC for Titan operations.

The author appreciates the contribution of Christine Boykin at Johnson Space Center to the choice of the height levels and time periods selected for study. Gregory Taylor of ENSCO, Inc. (AMU), directed my attention to the paper of Carr et al., which contained the small median test used herein. Wade Batts of Marshall Spaceflight Center supplied the climatological data used to select the small median parameters. Gregory Taylor and Timothy Wilfong provided thorough reviews of the first draft of this paper. Their thoughtful comments made this a better paper as did those of several anonymous reviewers. The author appreciates the assistance of Ann Yersavich, who prepared the manuscript for publication. 


\section{REFERENCES}

Aitchison. J., and J. C. Brown. 1966: The Lognormal Distribution. Cambridge University Press. $176 \mathrm{pp}$

Carr. F. H. P. L Spencer C A Doswell III, and J. D. Powell 1995: A comparison of two objective analysis techniques for profiler time-height data. Mon. Wea. R''́. 123, 2165-2180.

Douglas. M. W. and D. J. Stensrud. 1996: Upgrading the North American upper-air observing network: What are the possibilities? Bull. Amer. Meteror. Soc., 77, 907-924.

Mecham, M.. 1995: Wind shear blamed in Apstar-2 loss. Aviat. Weeh Space Terhmol., 143, 24.

Merceret. F. J. 1976: Measuring atmospheric turbulence with airborne hot-film inemometers. J. Appl. Meteor. 15, 482-490)

Nastrom. G. D., and F. D. Eaton, 1995: Variations of winds and turbulence seen by the $50-\mathrm{MH}$. radar at White Sands Missile Range, New Mexico. J. Appl. Meteor. 34, 21.35-2148.

NOAA. 1994: Wind Profiler Assessment Report. NOAA. 141 pp. A Available from National Weather Service. Office of Meteorology W/OM22, 1325 East-West Highway. Silver Spring. MD $20910-3233.1$
Ottersten, H., 1969: Atmospheric structure and radar backscattering in clear air. Rudio Sci., 4, 1179-1193.

Schumann, R. K. Atchison, G. E. Taylor, J. Warburton, M. M. Wheeler, and A. Yersavich, 1993: Software requirements specification for the new wind algorithm in NASAs $50 \mathrm{MHz}$ Doppler radar wind profiler. 3 ] pp. [Available from ENSCO. Inc., 445 Pineda Court. Melhourne, FL 32940.1

Spencer. P. L.. F. H. Carr, and C. A. Doswell III, 1996: Diagnosis of an amplifying baroclinic wave using wind profiler data. Mon. Wea. Rev'., 124, 209-223.

Siull, R. B., 1989: An Introduction to Boundary Layer Meteorology. Kluwer Academic Publishers, 666 pp.

Vinnichenko, N. K.. 1970: The kinetic energy spectrum in the free atmosphere. Tellus, XXIl, 158-166.

, and J. A. Dutton, 1969: Empirical studies of atmospheric structure and spectra in the free almosphere. Radio $S_{c i}, 4,1115$ 1126.

Wilfong. T., S. Smith, and R. Creasey, 1993: High temporal resolution velocity measurements from a wind profiler. J. Spacecr. Rockets. 30, $348-354$. 
